\title{
Androgen receptor is overexpressed in boys with severe hypospadias, and ZEB1 regulates androgen receptor expression in human foreskin cells
}

\author{
Liang Qiao', Gregory E. Tasian², Haiyang Zhang ${ }^{3}$, Mei Cao ${ }^{3}$, Max Ferretti ${ }^{3}$, Gerald R. Cunha ${ }^{3}$ and Laurence S. Baskin ${ }^{3}$
}

INTRODUCTION: ZEB1 is overexpressed in patients with severe hypospadias. We examined the interaction between ZEB1 and the androgen receptor (AR) in vitro and the expression of $A R$ in boys with hypospadias.

RESULTS: ZEB1 and AR colocalize to the nucleus. Estrogen upregulated ZEB1 and AR expression. Chromatin immunoprecipitation (ChIP) demonstrated that ZEB1 binds to an E-box sequence in the AR gene promoter. AR expression is higher in subjects with severe hypospadias than those with mild hypospadias and control subjects $(P<0.05)$. ZEB1 physically interacts with AR in human foreskin cells.

DISCUSSION: AR is overexpressed in patients with severe hypospadias. Environmental estrogenic compounds may increase the risk of hypospadias by facilitating the interaction between ZEB1 and AR.

METHODS: $\mathrm{Hs} 68$ cells, a fibroblast cell line derived from neonatal human foreskin, were exposed to 0, 10, and $100 \mathrm{nmol} / \mathrm{l}$ of estrogen, after which the cellular localization of ZEB1 and AR was assessed using immunocytochemistry. To determine if ZEB1 interacted with the AR gene, ChIP was performed using ZEB1 antibody and polymerase chain reaction (PCR) for AR. Second, AR expression was quantified using real-time PCR and western blot in normal subjects $(n=32)$, and subjects with mild $(n=16)$ and severe hypospadia $(n=16)$.

$\mathbf{H}$ ypospadias is a common congenital genitourinary anomaly that affects $\sim 1: 125$ live male births (1). Hypospadias has been associated with aberrant androgen signaling during development $(2,3)$. Additionally, boys who were exposed to elevated levels of estrogen in utero have a higher incidence of hypospadias $(4,5)$. While the role of androgen disruption in the pathogenesis of hypospadias is supported by these studies, the molecular mechanisms underlying the association between estrogen exposure, androgen signaling, and hypospadias are poorly understood.

$Z E B 1$, a zinc finger box gene that acts as a transcriptional regulator, is estrogen-responsive and is overexpressed in patients with severe hypospadias (6-8). ZEB1 has been heavily studied as protein that is involved in cancer progression. These studies have shown that ZEB1 decreases in intercellular adhesion molecules such as E-cadherin (9-12). Based on previous studies which have demonstrated that ZEB1 is overexpressed in patients with severe hypospadias, we have hypothesized that zeb1 overexpression decreases cellular adhesion in the developing male urethra and ventral penile skin, which could contribute to the abortive penile development seen in hypospadias (8).

Androgen receptor (AR) and $\mathrm{ZEB} 1$ have been shown to reciprocally regulate each other in a breast cancer cell line known to be androgen-responsive (13). We hypothesize that $\mathrm{ZEB} 1$ regulates the transcription of $\mathrm{AR}$ in penile development and that the ZEB1 signaling pathway mediates the effects of estrogen in the developing penis, which could be important in the pathogenesis of hypospadias. This study determined the effects of estrogen on ZEB1 and AR expression in a cell line derived from neonatal human foreskin and ascertained how ZEB1 interacts with AR in vitro. We determined expression levels of AR in normal boys and those with hypospadias.

\section{RESULTS}

ZEB1 and AR Colocalize to the Nucleus

Immunocystochemistry demonstrate that ZEB1 and AR colocalize to the nuclei of Hs68 cells. Nuclear localization was seen both with and without estrogen supplementation. Increasing estrogen concentration increased expression of both ZEB1 and AR (Figure 1). AR is expressed in the stratum basale of preputial skin in normal subjects and those with hypospadias (Figure 2). In Hs68 cells, real-time polymerase chain reaction (PCR) demonstrated that $A R$ mRNA increases as estrogen concentration increases (Figure 3 ).

\section{ZEB1 Regulates AR by Binding to AR Promoter}

As previously reported, the putative E-box sequence (CAGGTG) found at nucleotides -117 to -123 of the AR gene is a binding site for ZEB1 (13). In the chromatin immunoprecipitation (ChIP) assay performed on Hs68 cells, we found, 


\section{Articles | Qiao et al.}
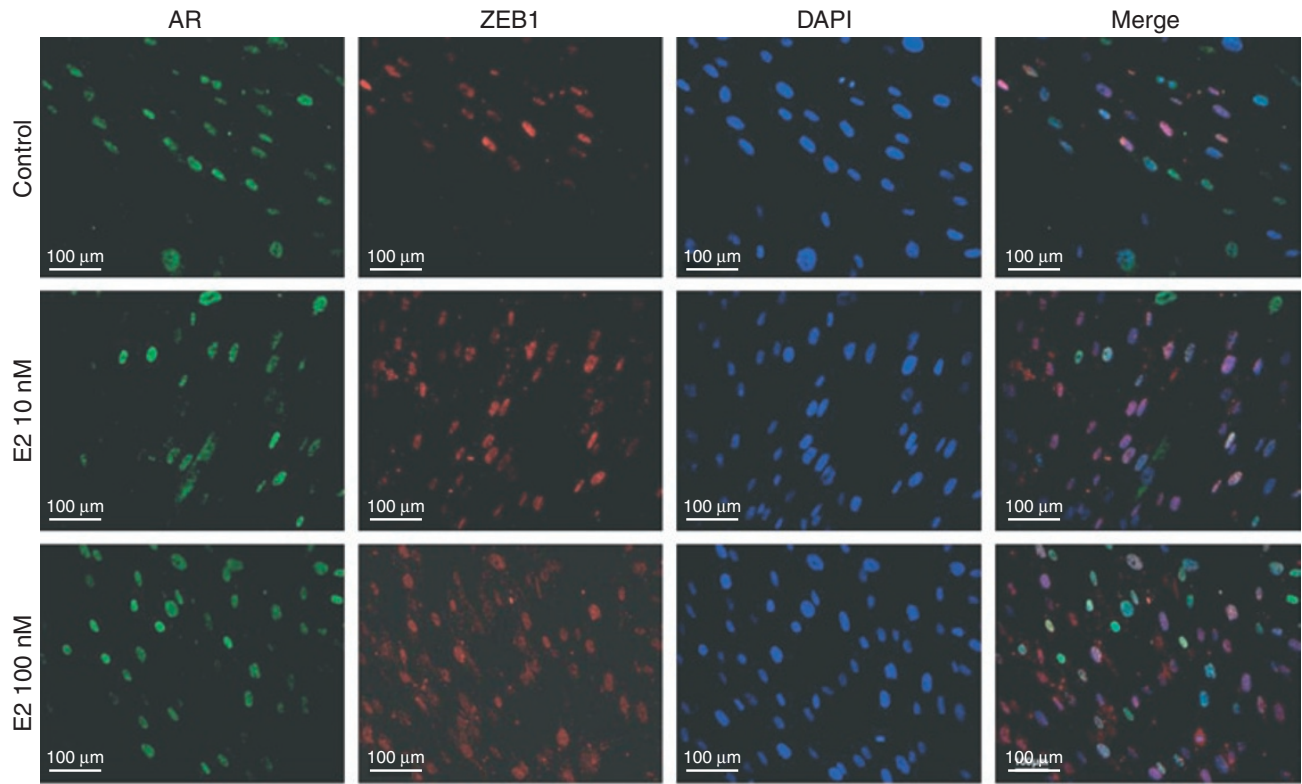

Figure 1. ZEB1 and androgen receptor are expressed in the nucleus of Hs68 cells. ZEB1 and AR colocalize to the nuclei of Hs68 cells. Estrogen (E2) increases the expression of both ZEB1 and AR. Original magnification $\times 200$. AR, androgen receptor.

a

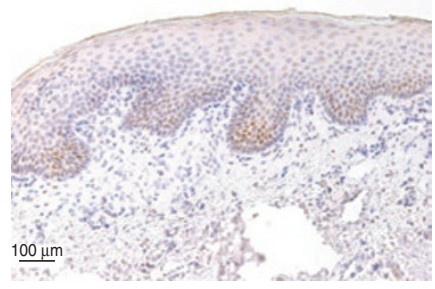

b

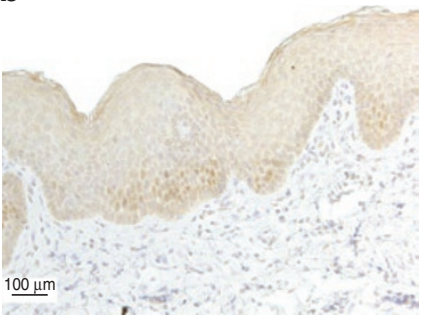

Figure 2. AR is expressed in the stratum basale of preputial skin. In both (a) normal subjects and (b) those with hypospadias, AR is expressed in the stratum basale of preputial skin. Original magnification $\times 200$. $A R$, androgen receptor.

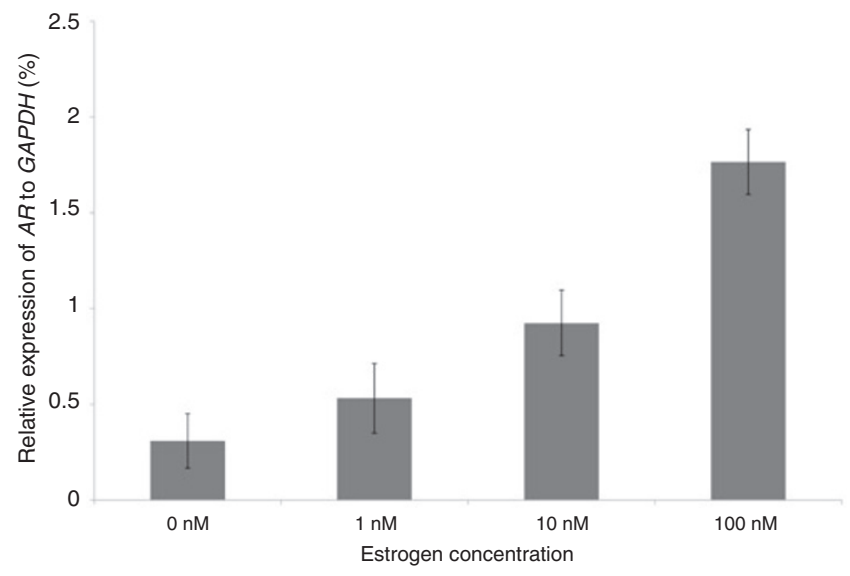

Figure 3. Estrogen increases $A R$ expression in vitro. AR mRNA expression in $\mathrm{Hs} 68$ cells increases with increasing estrogen concentration. $A R$, androgen receptor.

after amplification with PCR, $A R$ fragments were precipitated by the anti-ZEB1 antibody. $A R$ fragments were not seen in the negative control IgG antibody (Figure 4). a

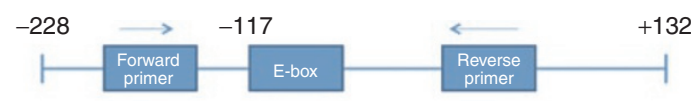

tagcagggca gatcttgtcc accgtgtgtc ttcttctgca cgagactttg aggctgtcag $\longrightarrow$ E-box agcgcttttt gegtggttgc tccogcaagt ttccttctct ggagcttcce gcaggtgggc agctagctgc agcgactacc gcatcatcac agcctgttga actcttctga gcaagagaag gggaggcggg gtaagggaag taggtggaag attcagccaa gctcaaggat ggaagtgcag $\overleftrightarrow{\longleftrightarrow \text { ttagggctgg gaagggtcta ccctcggccg ccgtccaaga cctaccgagg agctttccag }}$ aatctgttcc agagcgtgcg cgaagtgatc cagaacccgg gccccaggca cccagaggcc

b

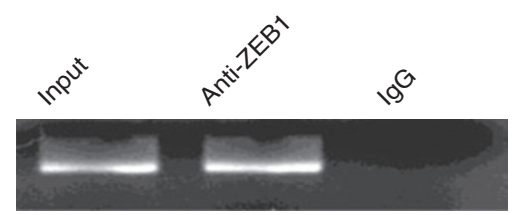

Figure 4. ZEB1 binds to E-box domain region of the AR gene promoter. $\mathrm{AR}$ and ZEB1 physically interact in $\mathrm{Hs} 68$ cells. (a) Schematic of potential E-box located in the AR promoter. (b) ChIP analysis demonstrates that ZEB1 binds the AR promoter. The input sample is the positive control and IgG is the negative control. AR, androgen receptor; ChIP, chromatin immunoprecipitation.

\section{AR Is Overexpressed in Patients With Severe Hypospadias}

The mean expression of $A R$ mRNA relative to GAPDH in control subjects and those with mild and severe hypospadias was 15.26, 21.33, and 87.37, respectively (Figure 4). There was no difference in the $A R$ expression levels between control and subjects with mild hypospadias; however, there were statistically significant differences in expression of $A R$ between subjects with severe hypospadias and control subjects $(P<0.001)$ and between subjects with severe and mild hypospadias $(P<0.001)$. 
a

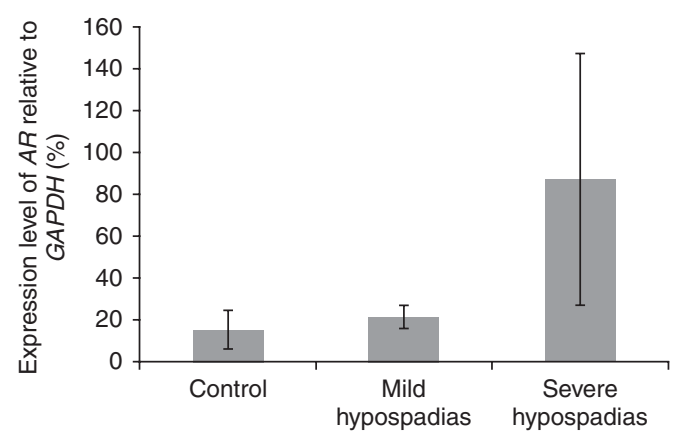

b

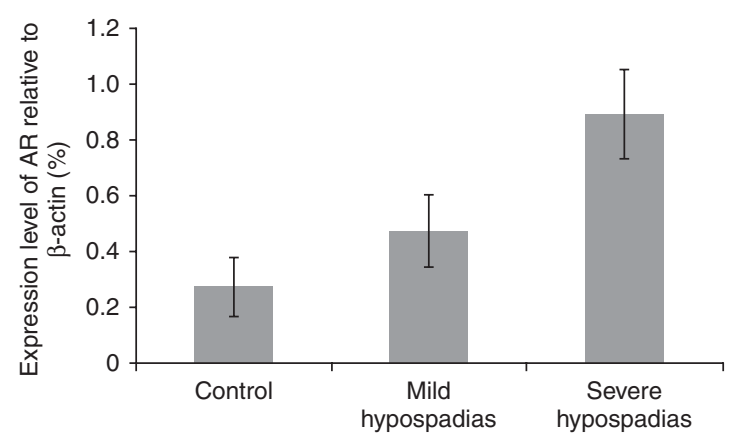

C

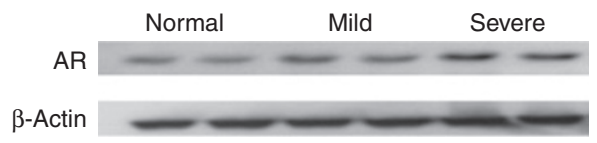

Figure 5. AR is overexpressed in patients with severe hypospadias. AR is overexpressed in subjects with severe hypospadias at both the (a) mRNA and $(\mathbf{b}, \mathbf{c})$ protein levels. AR, androgen receptor.

The expression levels of AR protein relative to $\beta$-actin in control subjects and those with mild and severe hypospadias were $0.27,0.47$, and 0.89 . Expression of AR at the protein level was significantly higher in subjects with severe hypospadias compared to controls and those with mild hypospadias (both $P<0.01$ ) (Figure 5). Although AR expression levels were higher in those with mild hypospadias than control subjects, this difference approached but did not achieve statistical significance $(P=0.05)$.

\section{DISCUSSION}

Androgen signaling through the AR is critical for normal penile development. Diminished androgen signaling results in a spectrum of incompletely virilized external genitalia: complete androgen insensitivity results in external genitalia with a female phenotype and partial insensitivity results in ambiguous genitalia of varying degrees. Originally put forth by Alfred Jost, it has long been held that the female genitalia phenotype is the "default" pathways that occurs in the absent of androgen signaling. Recent studies have refined the Jost hypothesis and suggest that penile development is a balance of androgenic and estrogenic activity (14). Although the cause of most cases of hypospadias is unknown, disruption of normal androgen signaling is thought to have a significant role. Estrogen is a potential disruptor of androgen signaling as epidemiologic studies have shown that boys who were exposed to elevated levels of estrogen in utero have increased risk of hypospadias (4).

This study has shown that AR is overexpressed in patients with severe hypospadias. We have also demonstrated that ZEB1 binds to the E-box domain of the AR promoter in human foreskin fibroblasts. It has previously been shown that estrogen increases both mRNA and protein levels of ZEB1 and that ZEB1 is overexpressed in patients with severe hypospadias (8). Graham has shown that ZEB1 binds to the E-box domain on the AR promoter in breast cancer cell lines (13). The interaction of ZEB1 and the AR reporter in widely disparate models suggests that ZEB1 upregulation of AR is a preserved molecular mechanism through which androgen signaling is regulated. However, to our knowledge, no other studies have examined the role of ZEB1 in androgen signaling.

ZEB1 has been extensively studied as a factor involved in epithelial to mesenchymal transition. It is a key factor in cancer progression during which epithelial cells lose expression of the intercellular molecule E-cadherin and thus become migratory and able to invade. ZEB1 is also a critical molecule during embryogenesis as it is expressed throughout the developing embryo and is necessary for survival (15). This study has shown that $\mathrm{ZEB} 1$ binds to the AR promoter. We previously reported that estrogen increases ZEB1 expression and that ZEB1 is overexpressed in boys with severe hypospadias. Both ZEB1 and AR are expressed in the stratum basale of preputial skin. These results suggest that estrogen, through ZEB1, upregulates AR expression in patients with severe hypospadias.

Estrogen-driven ZEB1 upregulation of AR may provide additional understanding of how hypospadias develops. Hypospadias may arise from abnormal developmental events occurring either during hormone-independent formation of the ambisexual genital tubercle or subsequent hormonedependent sex differentiation. Environmental exposure to estrogens is widespread and multiple estrogen-responsive genes (ATF3, CTGF, CY61, GADD45b) have been shown to be upregulated in patients with hypospadias relative to subjects with normal genitalia (7). It is estimated that 50 million women take oral contraceptives (OCPs); $3-4 \%$ continue taking OCPs inadvertently after becoming pregnant (16). It is unknown what effect this supraphysiologic dose of estrogen may have on the developing fetus; however, it is known that women who maintain soy-based diets, which are rich in phytoestrogens, during pregnancy have increased risk for having a male child with hypospadias (17). Our finding that ZEB1, which is known to be estrogen-responsive, interacts directly with the AR raises the hypothesis that ZEB1 may be an important mediator of estrogen-driven aberrant androgen signaling in hypospadias.

In our study, AR expression levels increased in Hs68 cells as estrogen concentration increased and AR expression was higher in preputial skin of boys with severe hypospadias compared to control subjects and those with mild hypospadias. 
It seems counter-intuitive that AR would be upregulated in subjects with hypospadias, which would seem to be associated with decreased rather than overactive androgen signaling. However, male mice exposed prenatally to medroxyprogesterone, a synthetic progesterone, develop hypospadias and have elevated levels of AR (18). However, neonatal exposure to high levels of estrogen downregulates AR in the testes, prostate, and seminal vesicles, with increasing concentrations of estrogen resulting in corresponding lower levels of AR expression (19-21).

One explanation is that estrogen has differential effects on AR expression depending on tissue type and local environment. Both Hs68 cells and the human subject preputial tissue used in this study are derived from ectoderm, which may have a different response to estrogen stimulation than the mesoderm-derived gonads, prostate, and seminal vesicles. Another explanation is that AR expression is dependent on the timing of estrogen exposure. The studies that reported decreased AR expression after administration of estrogen all used rodent animal models exposed to estrogen after birth (19-21). Hypospadias, however, occurs early in gestation as penis development occurs between 9 and $13 \mathrm{wk}$. It is possible that postnatal estrogen exposure has a suppressive effect on AR expression while prenatal exposure may increase $\mathrm{AR}$, at least in preputial skin.

We believe the most likely explanation for the findings is that there are likely multiple points of interaction between AR-induced signaling and other sex steroid pathways during genital development. In mouse models, AR, estrogen receptor, and progesterone receptors are differentially expressed in the developing mouse genital tubercle and are responsive to exogenous estrogen (22-24). Additionally, many of the environmental and pharmacologic compounds that have been associated with hypospadias signal through estrogen receptor and progesterone receptor in addition to or independent of AR (18,24-32). The multiple points through which estrogenic compounds interact in the sex hormone signaling pathways involved in penile development and the emerging evidence that both androgens and estrogens are involved in male genitalia development strengthen the theory that estrogen-induced androgen signaling disruption is a significant molecular mechanism underlying hypospadias.

There are limitations of the Hs68 cell line used in this study. Because Hs68 cells are derived from the foreskin of a human male neonate, they might not reflect the gene expression profile or cellular properties of the developing urethral plate, which is derived from endoderm. Also, Hs68 cells are fibroblasts; hence, we cannot definitively state that estrogen increases ZEB1 expression in human foreskin epithelium in vitro. However, because ZEB1 levels vary by tissue type, we felt it was of primary importance to perform the in vitro studies on cells that originated from human neonatal male genital skin (33). Furthermore, previous studies in which Hs68 cells were exposed to estrogen have supported findings in human tissue samples and mouse models, which is also what we observed in this study (34).

Our findings that estrogen upregulates $A R$ in vitro and that $\mathrm{AR}$ is overexpressed in patients with severe hypospadias suggest that estrogen-induced abnormal AR expression mediated by ZEB1 may be a mechanism through which estrogen contributes to the development of hypospadias. However, given the complexity of sex steroid signaling involved in penile development, we cannot attribute a single receptor or signaling pathway to be the only factor involved in hypospadias pathogenesis. The interaction of estrogen and progesterone pathways with AR signaling in hypospadias should be further explored. It remains unknown if ZEB1 interacts with these pathways.

\section{METHODS}

\section{Subject Characteristics}

Preputial tissue was obtained from 32 male subjects with hypospadias undergoing surgical repair at the University of California, San Francisco (UCSF), between 2005 and 2010. The degree of hypospadias was determined by the position of the urethral meatus and was classified as mild (meatus at or distal to the mid-shaft of the penis, $n=$ 16 ) or severe (meatus proximal to the mid-shaft of the penis, $n=16$ ). Normal preputial skin was obtained from subjects $(n=14)$ undergoing circumcision during the same time period. All subjects were prospectively enrolled, and informed consent was obtained from parents/ legal guardians prior to the operation. This study was approved by the UCSF Committee on Human Research.

\section{RNA Preparation, Reverse Transcription, and Real-Time PCR}

We used the protocols previously described (8). In brief, RNA was isolated with RNeasy Midi Kit (QIAGEN, Valencia, CA), and its quality and purity was measured by NanoDrop spectrophotometer (Thermo Fisher Scientific, Wilmington, DE). RNA integrity was visualized by the sharpness of the $28 \mathrm{~s}$ and 18 s ribosomal RNA bands in agarose gels. After standard reverse transcription, the real-time PCR primers were designed according to target gene sequence published on PubMed and were synthesized by Integrated DNA Technologies (San Diego, CA) (Table 1).

SYBR Green Real-time quantitative PCR (Applied Biosystems, Foster City, CA) was performed using a 7300 fast sequence detection system according to the manufacture's instruction and the Minimum Information for Publication of Quantitative Real-Time PCR Experiment (MIQE) guidelines (35). Primer titration and dissociation experiments were performed so that no primer dimers or false amplifications would interfere with the result. Cycle threshold $(\mathrm{Ct})$ number was extracted for both the reference (GADPH) and target genes with auto baseline and manual thresholds. PCR was repeated three times for each sample. Expression levels of $A R$ are reported relative to GAPDH using two delta-delta cycle threshold (36). There was no difference in the amplification kinetics of $G A D P H$ and $A R$.

\section{Protein Isolation and Western Blot}

Protein from the patient samples was extracted with NE-PER Nuclear and Cytoplasmic extraction reagents kit (Pierce Biotechnology, Rockford, IL). Protease inhibitors (Thermo scientific halt protease inhibitor cocktail) were added to the extraction reagents. Cell lysates containing $20 \mu \mathrm{g}$ of protein were loaded into a $10 \%$ Precast SDS-PAGE $\mathrm{Gel}$, followed by electrophoresis and transfer of the proteins onto a

Table 1. PCR primer sequences of androgen receptor and GADPH

\begin{tabular}{llc}
\hline Gene & Primer sequence & Product size (bp) \\
\hline$A R$ & F: AAGACGCTTCTACCAGCTCACCAA & 170 \\
& R: TCCCAGAAAGGATCTTGGGCACTT & \\
GAPDH & F: CATGTTCGTCATGGGTGTGAACCA & 160 \\
& R:AGTGATGGCATGGACTGTGGTCAT & \\
\hline
\end{tabular}

$A R$, androgen receptor. 
polyvinylidene fluoride membrane, which was stained with Ponceau $\mathrm{S}$. Detection of target proteins was performed with an electrochemiluminescence kit (Amersham Life Science, Arlington Heights, IL) using primary antibodies for AR (ab77557; Abcam, Cambridge, MA, 1:100) and $\beta$-actin (Sigma, A5441, 1:3000 dilution). After secondary antibody hybridization, the images were analyzed with ChemiImager4000 (Alpha Innotech, San Leandro, CA). Expression levels of AR are reported relative to $\beta$-actin.

\section{Cell Culture}

Hs68 cells, a fibroblast cell line derived from the foreskin of a normal human neonate, (American Type Culture Collection, Manassas, VA), were cultured in Dulbecco's modified Eagle's medium supplemented with $10 \%$ fetal bovine serum, $1 \%$ nonessential amino acid, 10,000 unit $/ \mathrm{ml}$ penicillin, $10,000 \mathrm{mcg} / \mathrm{ml}$ streptomycin $\mathrm{SO}_{4} 0.025 \mathrm{mg} / \mathrm{ml}$ fungizone, and $110 \mu \mathrm{g} / \mathrm{ml}$ sodium pyruvate at $37^{\circ} \mathrm{C}$ in $5 \% \mathrm{CO}_{2}$ until $80 \%$ confluence. Cells used in the experiments were from passages 5 through 10 . At the time of experiment, cells were starved of fetal bovine serum for $12 \mathrm{~h}$ and subsequently treated with estrogen ( $\beta$-Estradiol-Water Soluble; Sigma, St Louis, MO) at 0, 10, and $100 \mathrm{nmol} / \mathrm{l}$ for $8 \mathrm{~h}$.

\section{Immunohistochemistry}

Preputial skin samples were fixed in formalin, paraffin embedded, and sectioned $(5 \mu \mathrm{m})$. Antigens were retrieved using antigen unmasking solution (Vector Laboratory, Burlingame, CA). After blocking, slides were incubated overnight at $4{ }^{\circ} \mathrm{C}$ with an affinity purified rabbit polyclonal anti-AR antibody (PG21-42, gift of Lynn Birch, University of Illinois at Chicago). Staining of the tissue was performed with the Elite ABC kit (Vector Laboratory) followed by hematoxylin counterstain.

\section{Immunofluorescence Staining}

After treatment with estrogen as described above, the Hs68 cells were fixed with ice-cold methanol, permeabilized with $0.05 \%$ Triton X-100, and blocked with Superblock (Perbio Science GmbH, Heidelberg, Germany). The cells were then incubated with goat antiZEB1 antibody (R17; Santa Cruz Biotechnology, Santa Cruz, CA) and rabbit anti-AR antibody (gift of Gail Prins, University of Illinois at Chicago) overnight at $4{ }^{\circ} \mathrm{C}$ followed by incubation with donkey anti-rabbit Alexa Fluor 488 FITC conjugated antibody for $1 \mathrm{~h}$ at room temperature, and donkey anti-goat Alexa Fluor 594 FITC conjugated antibody for $1 \mathrm{~h}$. The cells were stained with 4',6-diamidino2-phenylindole (DAPI) to stain the nuclei and then photographed using a LEICA DM 4000B fluorescence microscope and a LEICA DFC 500 digital camera.

\section{ChIP Assay}

The ChIP assay was performed by using the EZ-Magna ChIP G kit (Upstate, No.17-409, Millipore, Billerica, MA) following the manufacturer's instructions. HS68 cells were grown to $80-90 \%$ confluence after which formaldehyde was added to the culture medium to a final concentration of $1 \%$ for $10 \mathrm{~min}$. The unreacted formaldehyde was quenched by incubating with $10 \times$ glycine buffer and then the cells were rinsed and resuspended in phosphate-buffered saline with protease inhibitor cocktail II. Cell and nuclear lysis buffer were added followed by sonication of cell lysate and centrifugation at $4^{\circ} \mathrm{C}$ for $10 \mathrm{~min}$. Supernatants were collected and diluted 1:10 with dilution buffer. A portion of diluted supernatant (1\%) was kept to estimate the amount of DNA present in different samples; this is referred to as "input" sample. Immunoprecipitation was carried out overnight at $4{ }^{\circ} \mathrm{C}$ by adding the immunoprecipitating antibody and $20 \mu \mathrm{l}$ fully suspended protein $\mathrm{G}$ magnetic beads. The antibodies used were rabbit polyclonal ZEB1 (2 $\mu \mathrm{g})$ (R17, sc-10573, Santa Cruz Biotechnology). The "input" sample and normal rabbit IgG $(1 \mu \mathrm{g})$ were also included as the positive and negative controls, respectively. After washing the protein $\mathrm{G}$ bead-antibody/chromatin complex, ChIP elusion buffer with proteinase $\mathrm{K}$ was added and incubated at $62^{\circ} \mathrm{C}$ for $2 \mathrm{~h}$. DNA was recovered and purified with DNA spin columns. After ChIP assays, routine PCR was used to quantify the DNA in the samples. The AR primers used for ChIP are as follows: forward 5'-GCG TGG TTG CTC CCG CAA G-3', reverse 5'-GGG TAG ACC CTT CCC AGC CC-3'.

\section{Statistical Analysis}

The difference of mRNA and protein expression levels of AR between controls, subjects with mild hypospadias, and subjects with severe hypospadias was determined using ANOVA with Bonferroni correction. Statistical analysis was performed with SPSS Statistic 17 (IBM, Chicago, IL)

\section{ACKNOWLEDGMENT}

L.Q. and G.E.T. conducted the work while at the University of California, San Francisco, Department of Urology.

\section{STATEMENT OF FINANCIAL SUPPORT}

This study was supported by NIH grant R01 DK058105.

\section{REFERENCES}

1. Paulozzi LJ. International trends in rates of hypospadias and cryptorchidism. Environ Health Perspect 1999;107:297-302.

2. Gray LE Jr, Wolf C, Lambright C, et al. Administration of potentially antiandrogenic pesticides (procymidone, linuron, iprodione, chlozolinate, $\mathrm{p}, \mathrm{p}^{\prime}$-DDE, and ketoconazole) and toxic substances (dibutyl- and diethylhexyl phthalate, PCB 169, and ethane dimethane sulphonate) during sexual differentiation produces diverse profiles of reproductive malformations in the male rat. Toxicol Ind Health 1999;15:94-118.

3. Aschim EL, Nordenskjöld A, Giwercman A, et al. Linkage between cryptorchidism, hypospadias, and GGN repeat length in the androgen receptor gene. J Clin Endocrinol Metab 2004;89:5105-9.

4. Klip H, Verloop J, van Gool JD, Koster ME, Burger CW, van Leeuwen FE; OMEGA Project Group. Hypospadias in sons of women exposed to diethylstilbestrol in utero: a cohort study. Lancet 2002;359:1102-7.

5. Brouwers MM, Feitz WF, Roelofs LA, Kiemeney LA, de Gier RP, Roeleveld N. Risk factors for hypospadias. Eur J Pediatr 2007;166:671-8.

6. Garavelli L, Cerruti-Mainardi P, Virdis R, et al. Genitourinary anomalies in Mowat-Wilson syndrome with deletion/mutation in the zinc finger homeo box 1B gene (ZFHX1B). Report of three Italian cases with hypospadias and review. Horm Res 2005;63:187-92.

7. Wang Z, Liu BC, Lin GT, et al. Up-regulation of estrogen responsive genes in hypospadias: microarray analysis. J Urol 2007;177:1939-46.

8. Qiao L, Tasian GE, Zhang H, Cunha GR, Baskin L. ZEB1 is estrogen responsive in vitro in human foreskin cells and is over expressed in penile skin in patients with severe hypospadias. J Urol 2011;185: 1888-93.

9. Ozaki C, Obata S, Yamanaka H, Tominaga S, Suzuki ST. The extracellular domains of E- and N-cadherin determine the scattered punctate localization in epithelial cells and the cytoplasmic domains modulate the localization. J Biochem 2010;147:415-25.

10. Takeichi M. Morphogenetic roles of classic cadherins. Curr Opin Cell Biol 1995;7:619-27.

11. Drake JM, Strohbehn G, Bair TB, Moreland JG, Henry MD. ZEB1 enhances transendothelial migration and represses the epithelial phenotype of prostate cancer cells. Mol Biol Cell 2009;20:2207-17.

12. Kakihana $\mathrm{M}$, Ohira $\mathrm{T}$, Chan $\mathrm{D}$, et al. Induction of $\mathrm{E}$-cadherin in lung cancer and interaction with growth suppression by histone deacetylase inhibition. J Thorac Oncol 2009;4:1455-65.

13. Graham TR, Yacoub R, Taliaferro-Smith L, et al. Reciprocal regulation of ZEB1 and AR in triple negative breast cancer cells. Breast Cancer Res Treat 2010;123:139-47.

14. Yang JH, Menshenina J, Cunha GR, Place N, Baskin LS. Morphology of mouse external genitalia: implications for a role of estrogen in sexual dimorphism of the mouse genital tubercle. J Urol 2010;184(4 Suppl): 1604-9.

15. Miyoshi T, Maruhashi M, Van De Putte T, Kondoh H, Huylebroeck D, Higashi Y. Complementary expression pattern of Zfhx1 genes Sipl and deltaEF1 in the mouse embryo and their genetic interaction revealed by compound mutants. Dev Dyn 2006;235:1941-52. 
16. Thayer KA, Ruhlen RL, Howdeshell KL, et al. Altered prostate growth and daily sperm production in male mice exposed prenatally to subclinical doses of 17alpha-ethinyl oestradiol. Hum Reprod 2001;16:988-96.

17. North K, Golding J. A maternal vegetarian diet in pregnancy is associated with hypospadias. The ALSPAC Study Team. Avon Longitudinal Study of Pregnancy and Childhood. BJU Int 2000;85:107-13.

18. Willingham E, Agras K, de Souza AE Jr, et al. Steroid receptors and mammalian penile development: an unexpected role for progesterone receptor? J Urol 2006;176:728-33.

19. McKinnell C, Atanassova N, Williams K, et al. Suppression of androgen action and the induction of gross abnormalities of the reproductive tract in male rats treated neonatally with diethylstilbestrol. J Androl 2001;22:323-38.

20. Prins GS, Birch L. The developmental pattern of androgen receptor expression in rat prostate lobes is altered after neonatal exposure to estrogen. Endocrinology 1995;136:1303-14.

21. Williams K, Fisher JS, Turner KJ, McKinnell C, Saunders PT, Sharpe RM. Relationship between expression of sex steroid receptors and structure of the seminal vesicles after neonatal treatment of rats with potent or weak estrogens. Environ Health Perspect 2001;109:1227-35.

22. Agras K, Willingham E, Liu B, Baskin LS. Ontogeny of androgen receptor and disruption of its mRNA expression by exogenous estrogens during morphogenesis of the genital tubercle. J Urol 2006;176(4 Pt 2): 1883-8.

23. Agras K, Willingham E, Shiroyanagi Y, Minasi P, Baskin LS. Estrogen receptor-alpha and beta are differentially distributed, expressed and activated in the fetal genital tubercle. J Urol 2007;177:2386-92.

24. Agras K, Shiroyanagi Y, Baskin LS. Progesterone receptors in the developing genital tubercle: implications for the endocrine disruptor hypothesis as the etiology of hypospadias. J Urol 2007;178:722-7.

25. Yucel S, Cavalcanti AG, Wang Z, Baskin LS. The impact of prenatal androgens on vaginal and urogenital sinus development in the female mouse. J Urol 2003;170(4 Pt 1):1432-6.
26. Blaschko SD, Willingham EJ, Baskin LS. Embryonic exposure to low-dose 17beta-estradiol decreases fetal mass sex specifically in male mice and does not cause hypospadias. J Investig Med 2006;54:490-5.

27. Yucel S, Desouza A, Baskin LS. In utero prednisone exposure affects genital development. J Urol 2004;172(4 Pt 2):1725-30; discussion 1730.

28. Buckley J, Willingham E, Agras K, Baskin LS. Embryonic exposure to the fungicide vinclozolin causes virilization of females and alteration of progesterone receptor expression in vivo: an experimental study in mice. Environ Health 2006;5:4.

29. Hsieh MH, Grantham EC, Liu B, Macapagal R, Willingham E, Baskin LS. In utero exposure to benzophenone-2 causes hypospadias through an estrogen receptor dependent mechanism. J Urol 2007;178(4 Pt 2):1637-42.

30. Vilela ML, Willingham E, Buckley J, et al. Endocrine disruptors and hypospadias: role of genistein and the fungicide vinclozolin. Urology 2007;70:618-21.

31. Willingham E, Agras K, Vilela M, Baskin LS. Loratadine exerts estrogen-like effects and disrupts penile development in the mouse. J Urol 2006;175:723-6.

32. Kim KS, Torres CR Jr, Yucel S, Raimondo K, Cunha GR, Baskin LS. Induction of hypospadias in a murine model by maternal exposure to synthetic estrogens. Environ Res 2004;94:267-75.

33. Hurt EM, Saykally JN, Anose BM, Kalli KR, Sanders MM. Expression of the ZEB1 (deltaEF1) transcription factor in human: additional insights. Mol Cell Biochem 2008;318:89-99.

34. Liu B, Lin G, Willingham E, et al. Estradiol upregulates activating transcription factor 3, a candidate gene in the etiology of hypospadias. Pediatr Dev Pathol 2007;10:446-54.

35. Bustin SA, Benes V, Garson JA, et al. The MIQE guidelines: minimum information for publication of quantitative real-time PCR experiments. Clin Chem 2009;55:611-22.

36. Livak KJ, Schmittgen TD. Analysis of relative gene expression data using real-time quantitative PCR and the 2(-Delta Delta C(T)) Method. Methods 2001;25:402-8. 